\section{Diversidad e Inclusión en las Empresas de Moda}

Christian Vidal Beros ${ }^{(1)}$

Resumen: Se analiza la influencia del Soft Law en los mecanismos de autorregulación de las empresas y su aporte a la acción reguladora del Estado en ciertos temas. Se presenta la importancia de incorporar en los códigos de conducta o políticas corporativas, normas sobre promoción de la diversidad e inclusión y prohibición de discriminación, en particular en empresas de moda y/o retail.

Palabras claves: Soft Law - Autorregulación - Códigos de Conducta - Políticas corporativas - Diversidad - Inclusión - Derechos - Empresas de Moda - Política corporativa.

[Resúmenes en inglés y portugués en la página 92]

(1) Abogado, Licenciado en Derecho de la Pontificia Universidad Católica de Chile; Postgraduado en Derecho Público Global de la Universidad de Castilla-La Mancha, Toledo, España; cursó el Magíster en Derecho con mención en Derecho Público de la Universidad de Chile y es Master (C) en Derecho Digital y nuevas tecnologías por la Universidad de Salamanca. Profesor de las Facultades de Derecho de las Universidades Andrés Bello y Pedro de Valdivia, y de la Facultad de Economía y Negocios de la Universidad Finis Terrae.

\title{
Introducción
}

Desde hace un tiempo, los conceptos de diversidad e inclusión han dejado de ser parte del mundo de la sociología para trasladarse a otras ciencias sociales, no estando el Derecho, ajeno a ello. Desde un tibio reconocimiento desde la vereda de la "tolerancia" a fines de la década de los noventa del siglo pasado, al actual siglo, donde el simplemente "tolerar" ha dado paso a políticas de diversidad y pluralismo como fomento de la diferencia en la sociedad en general, y en empresas en particular.

El ordenamiento jurídico -como en la mayoría de los casos-, lamentablemente muchas veces llega tarde en reconocer realidades sociales vivas. Desde el punto de vista de los derechos fundamentales, es difícil encontrar constituciones que derechamente promuevan políticas sociales y jurídicas de inclusión, pluralismo y diversidad ${ }^{1}$. No obstante, de manera muy tímida, distintas leyes y constituciones, prohíben todo tipo de discriminación 
arbitraria y/o caprichosa tanto a nivel legislativo como al momento de aplicar la norma. ${ }^{2}$ Es justamente a nivel normativo, como consecuencia de la protección de trabajadores a través de procedimientos de tutela de derechos laborales, donde primeramente comenzó a consagrarse una prohibición expresa a la discriminación arbitraria, promoviendo la diversidad al interior de las distintas organizaciones. Es por ello, que el derecho laboral ha sido quien ha promovido de manera más tácita que expresa, la adopción por parte de las empresas, de políticas regulatorias y de autocontrol en relación con diversidad e inclusión. ${ }^{3}$ Veremos en el siguiente artículo -de manera breve pero lo más completa posible-, cómo la adopción de políticas corporativas sobre inclusión, diversidad y no discriminación en las empresas de moda, más que a una simple "moda", obedece a una preocupación del Derecho por crear una conciencia de autorregulación -más vinculada al soft law-, al interior de las organizaciones.

\section{Derecho, autoregulación y Soft Law}

Como otros autores ya lo han identificado, el mundo de la moda no posee un estatuto jurídico claro, ni menos legislativo, en relación al conjunto de relaciones jurídicas que al interior de las empresas, diseñadores, cadenas de retail y de toda la línea de producción pueda darse. Hasta no hace mucho tiempo, había consenso en que la única solución a una regulación global por parte del derecho a una industria transnacional como la moda, era asumida por el derecho internacional público y su propio sistema de fuentes. No obstante el nacimiento puntual que esta rama del derecho tuvo en su momento -principalmente para regular las relaciones entre Estados u organismos de derecho internacional público propiamente tal-, luego ha derivado en otras ramas del derecho que han surgido con autonomía, como por ejemplo el derecho internacional de los derechos humanos.

Sin embargo, como hoy entendemos la problemática global -incluso más allá de la industria de la moda, como puede ser el deporte, la normalización, la tributación y otros-, se complica pensar en una aplicación del derecho internacional público como una solución normativa eficiente. Es por lo anterior que el concepto de derecho global ha surgido hace un tiempo dentro de la discusión jurídica, como un área independiente y de aplicación propia dentro de la ciencia jurídica.

En efecto, este derecho global difiere en cuanto al sustento normativo de su homólogo "internacional público", en cuanto a fuentes y ámbito de aplicación. El derecho global no apunta a tratados o instrumentos de derecho estricto o hard law. Por el contrario, su esencia está constituida por principios, normas, estándares internacionales o regulación práctica de ciertos temas. No nos encontramos frente al resultado del Estado legisladorgenerador de normas, ni tampoco a tratados vinculantes.

Algunos autores han denominado soft law a este conjunto de principios, estándares internacionales, incluso recomendaciones, que muchas veces nacen a partir de la propia autorregulación de sujetos u organismos privados, creadores de redes transnacionales ${ }^{4}$. Producto de las normas de soft law podemos encontrar a la lex mercatoria ${ }^{5}$, la normativa ISO, los principios de gobierno corporativo o la lex sportiva ${ }^{6}$. ¿Difieren acaso todos los 
ejemplos de lo que podríamos aspirar en una regulación global -y judicial- a la industria de la moda? Creemos que no.

En efecto, cuando nos encontramos frente a prácticas que muchas veces dependen de la voluntad de adhesión de una determinada empresa, industria o gremio, resultando más complicado que el derecho positivo pueda ser receptor de dichos estándares o principios. Complicado, pero a la vez necesario, justamente por la voluntariedad en la que podemos encontrarnos hoy para que sean los propios actores de la industria quienes se autorregulen y obliguen a someterse a estándares y principios aceptados por todos.

Es por los ejemplos dados, que consideramos que la autorregulación en la industria de la moda puede constituir un principio de estándares aplicados a la industria para así transformarse en un derecho global, a modo de soft law. Efectivamente, la moda no es un fenómeno aislado y su globalización trae como consecuencia similares conflictos jurídicos en distintas partes del mundo; por otro lado, existen las voces que abogan por unificar criterios como los ya señalados: salud de los modelos, condiciones mínimas en cuanto a jornadas de trabajo, elementos de tributación uniforme y prácticas éticas en el cumplimiento normativo de las empresas de moda, por ejemplo. Sin embargo, son todos conflictos de derechos eventuales que igualmente deben en su momento, poder contar con un mecanismo de solución de controversias aplicado a esta realidad global, a modo de "justicia global" aplicada al mundo de la moda.

Tal como lo ha señalado innumerablemente la profesora Isabel Turégano, la doctrina del derecho apunta a una idea concreta de Justicia Global, Justicia Legal e Imperio de la Ley ${ }^{7}$. En efecto, es evidente la necesidad de imperio de un ordenamiento jurídico global que pueda hacer efectiva no sólo la responsabilidad de los estados u organismos en general, sino que también el cumplimiento de las resoluciones que condenen a dichas entidades frente a vulneraciones de derechos fundamentales de las personas. No obstante, lo anterior, surge una natural interrogante: ¿Cómo legitimar el orden político que haga efectiva la Justicia Global? Sobre este punto, consideramos que al ser la moda una industria con características muy particulares, dejar la solución de los conflictos a una jurisdicción común, implicaría lentitud en la solución de controversias como también la falta de conocimiento y especialización de los jueces que conozcan del asunto. De ahí la importancia de la autorregulación a través de principios, estándares y procedimientos propios a los cuales se sometan tanto los actores del gremio como los propios Estados. En definitiva, sebe atender a la creación de estructuras institucionales que respalden el actuar de la Justicia Global. Poco y nada podría funcionar un orden basado en principios de justicia distributiva y conmutativa sin la existencia de un orden legal consensuado por los actores globales.

Por lo anterior, la esperanza jurídica más efectiva para uniformar criterios resulta a mí parecer, las normas del soft law. Así lo señalan Bermúdez, Aguirre y Manasía (1991), que respecto al soft law “(...) algunos consideran que se trata de un Derecho Programa, basado en principios éticos que contribuyen a prefigurar lo que ulteriormente se desarrollará como Derecho y, cuyo significativo ejemplo son las resoluciones de los organismos internacionales" y a continuación agregan que:

Un sector de la doctrina considera que el soft law es un derecho espontáneo, pues nace o emerge de los usos y costumbres de los operadores jurídicos y 
comprende todo un conjunto de actos de variados tipos (guías jurídicas, leyes modelo, recomendaciones, resoluciones, entre otros), constituyendo un conjunto de reglas surgidas del obrar de los participantes en el contexto internacional.

Teniendo claridad respecto a que:

Los instrumentos de soft law se caracterizan por ser documentos que reflejan la tendencia actual de la comunidad internacional por una mayor interrelación, interdependencia y globalización. En este contexto las organizaciones internacionales, junto con otros actores internacionales, buscan mediante estos instrumentos promover acciones en materias de interés general en temas relativamente nuevos para la comunidad internacional en los cuales (por diferentes intereses políticos, económicos o de otra naturaleza) es difícil llegar a un acuerdo general con carácter obligatorio entre los Estados. Por ello, tales instrumentos no imponen obligaciones internacionales; sin embargo, esto no significa que carezcan de todo efecto jurídico. (Toro Huerta)

Coincidimos con lo planteado por los citados autores. Cualquier área productiva, temática, ya sea de la industria de la moda u otra semejante, debe configurar su propio sistema de principios, normas o estándares aceptados y validados por los actores del mismo, dando origen a un sistema integrado de principios y otorgando capacidad sancionatoria a determinados órganos encargados de dicha labor. Justamente este elemento final es el más complicado de implementar.

Indudablemente debemos pensar en una suerte de "pacto social de la moda", un contrato global de los actores de la industria, que delimite y someta -de manera voluntaria- a normas generales obligatorias, el actuar de la industria frente a derechos fundamentales de trabajadores, empresas y clientes, como asimismo la obligación de cumplir con ciertos estándares impositivos, medioambientales, laborales, aduaneros, marcarios o del derecho de consumo. Ese será justamente el desafío que el derecho de la moda tendrá en el tercer decenio del siglo XXI. Luego de su nacimiento y su actual posicionamiento como un área autónoma dentro de la ciencia jurídica, deberá ser capaz de cimentar un sistema de normas, principios y estándares que otorguen certeza a todos los intervinientes.

Habiendo entendido el desarrollo y avance normativo del soft law, conviene adentrarnos en la relevancia práctica para las empresas, de la autorregulación traducida en los códigos de conducta o políticas corporativas. Al respecto, señalan los profesores Juan Cuerva De Cañas y Manel Santilari Marnach en su curso del programa de Master en Abogacía Digital y Nuevas Tecnologías de la Universidad de Salamanca 2019-2020, que:

Si hace 20 años se hubiera preguntado en una clase de universidad qué es un código de conducta, las respuestas que habrían podido obtenerse serían de lo más variopinto. Actualmente, los códigos de conducta son una realidad y, además, una realidad que expande progresivamente su presencia en sectores, instituciones y empresas. Estas últimas, cada vez más, optan por autoimponer- 
se reglas de conducta (por ejemplo, medioambientales, sobre sostenibilidad, paridad de sexos y meritocracia, etc.) (...).

De acuerdo a lo anterior, cabe señalar que dentro de las características que los códigos de conducta o políticas corporativas internas de las empresas tienen, éstos en primer lugar no son de naturaleza legal. Es importante tener presente que como ya lo hemos advertido, las políticas corporativas no nacen del derecho estricto o hard law. La única intervención -indirecta-, que el derecho "duro" pueda tener en ellas, es que el legislador ordene que las empresas cuenten con políticas corporativas internas en determinadas áreas, principalmente para efectos de demostrar al regulador, su comportamiento autorregulatorio. No obstante, lo anterior, los códigos de conducta o políticas internas son parte del ya explicado soft law.

En segundo lugar, las políticas corporativas son obligatorias solamente en la medida que una empresa se haya adherido a ella, habiendo sido aprobada por el órgano de gobierno corporativo respectivo (pudiendo ser éste el directorio de la empresa, en la mayoría de los casos). Ahora bien, habiéndose adoptado por parte de una compañía una determinada política o código de conducta, la empresa es la primera obligada a su cumplimiento, principalmente porque ésta es el sujeto pasivo de respetar los principios en ella establecidos. Teniendo claro que las políticas corporativas no son leyes, estos códigos de conducta corporativos debieran ser fuente interna creadora de derecho "blando", es decir, un soft law corporativo, cuyo "piso normativo" sería lo señalado por la ley, tendiendo siempre la política corporativa a ir "más allá" del mínimo establecido por el legislador (hard law).

No obstante, el hecho de que sean postulados voluntarios a los cuales se someten las empresas en diversos temas, esto no hace que las políticas corporativas sean meras declaraciones de principios. Por el contrario, estos códigos deben establecer sanciones a la vulneración de los principios y derechos en ellos contenidos, justamente para que no nos encontremos frente a una mera declaración ética, sino que frente a un instrumento jurídico con características sancionatorias.

Como consecuencia de lo anterior, se debe definir la existencia de un órgano de control encargado de fiscalizar el cumplimiento de la política, para que éste no sea una mera declaración de principios éticos, algo ya señalado en los puntos precedentes. Será en el caso de la mayoría de las empresas -sean o no de moda-, el propio Directorio que aprobó la política, el que determine qué personas y/o gerencias dentro de la propia administración, tomen la responsabilidad de velar por la fiscalización y cumplimiento interno de la misma. Finalmente, y como en todo orden de normas obligatorias, el código de conducta debe tener VII) publicidad a sus destinatarios. Tal como ocurre con la publicidad de las normas, los destinatarios de las mismas deben tener conocimiento de sus disposiciones, lo cual en la práctica puede realizar la empresa publicando el texto de la política corporativa en su web interna (intranet) o externa, muros informativos o enviado por correo a todos los trabajadores. 


\section{Diversidad, inclusión y no discriminación en las empresas}

En el caso de las Políticas de Inclusión, Diversidad y No Discriminación, en adelante PIDND, éstas en el derecho chileno no son obligatorias legalmente, no obstante, su incorporación dentro del gobierno corporativo de la empresa, supone un mérito reputacional para aquellas compañías que las adopten. Lo anterior, por cuanto el objetivo de una PIDND, será el fomentar e impulsar el valor de la diversidad, aceptando el sello propio de cada persona, plasmando un compromiso de respeto por la diferencia, facilitando ambientes laborales inclusivos gestionados de manera responsable, con el fin de incorporar una fuerza de trabajo diversa que pueda comprender las necesidades de nuestros clientes, también diversos.

En ese orden de cosas, este tipo de políticas se aplica generalmente a todos los miembros no solo del gobierno corporativo de la empresa, sino que también a colaboradores y directores, clientes y proveedores, sin distinción de sexo, edad, condición social, religión, orientación sexual, género, raza, estado civil, sindicación, opinión política, discapacidad, nacionalidad, grupo étnico o cualquier otra condición protegida por la ley, y a todos los niveles jerárquicos de la compañía.

Lo anterior es consecuencia de la casuística. Desde hace no pocos años, los consumidores y los trabajadores han exigido mayor diversidad no solamente al interior de los espacios de trabajo -aceptando e incorporando trabajadores cuyas procedencias y realidades son distintas-, sino que también respecto a los productos y/o servicios ofrecidos por las empresas a sus consumidores directos. En relación a este segundo punto, y específicamente en casos de empresas de moda, los consumidores con el poder que hoy otorgan las redes sociales han sido enfáticos en exigir mayor diversidad de tallas, mayor diversidad en productos de cuidado personal para diferentes razas o tipos de cuerpos o incluso en eliminar sesgos de género al momento de definir si una determinada prenda deberá ser usada por un niño o una niña.

Profundizando lo señalado, toda política corporativa deberá velar por identificar las necesidades especiales de los clientes, impulsando iniciativas que permitan llegar a la mayor parte de ellos. Es así como deberán asumir el compromiso de darles la mejor atención y servicio, especialmente en materias de diversidad y no discriminación. Conocido es el ejemplo de dos trabajadoras afroamericanas de Amazon, las cuales notaron la escasez de productos para el cuidado capilar destinado a dicha comunidad: "Es un ejemplo de que si no cambias la perspectiva, si no sales de tu caja, no lo estás haciendo bien. ¿Cómo vamos a saber qué quiere el cliente si no sabemos cómo es? Lo importante es que la plantilla se parezca a sus propios clientes", resume Koro Castellano, que, como directora global de Diversidad e Inclusión, se encarga de que los aproximadamente 650.000 empleados de la tienda más grande del mundo estén bien repartidos en materia de género, origen, edad, identidad sexual y capacidades." ${ }^{18}$

Una empresa moderna deberá considerar la inclusión, diversidad y no discriminación como un activo, pues identificará en estos conceptos, líneas estratégicas de desarrollo, con potencial de crecimiento, en el corto, mediano y largo plazo. La responsabilidad corporativa con la diversidad, se fundamentará en las acciones comprometidas con sus colaboradores, clientes, proveedores, accionistas, la sociedad y su entorno en general, a través de sus compromisos corporativos y empresariales. 
Los principales lineamientos básicos que debiera tener toda PIDND son:

- Inclusión Laboral, entendida ésta como la búsqueda de un equipo de colaboradores de excelencia que reflejen los valores de la empresa y que sean referencia del mejor talento. Por esta razón es que la implementación de programas que propicien la inclusión laboral en diferentes sectores de la población como son las personas en situación de discapacidad o determinadas minorías o grupos vulnerables, sea tomado como un desafío por las compañías modernas.

- La diversidad como un valor intangible para toda empresa, por cuanto contar con equipos diversos hará de cualquier compañía un lugar más inclusivo y más productivo, aprovechando las potencialidades de trabajadores diferentes. Sin perjuicio de lo anterior, y tal como lo señalamos en el ejemplo de Amazon, contar con un crisol de trabajadores hará a una empresa más conocedora del mercado, de las necesidades diversas, de distintos grupos etáreos, sociales, étnicos y/o de gustos diversos, generando un plus reputacional y económico a largo plazo para la empresa.

- Equidad de género e igualdad salarial entre hombres y mujeres en lo que se refiere a empleabilidad laboral, formación, promoción profesional, condiciones de trabajo y remuneraciones, fomentando la equidad de género como manifestación de trato igualitario entre hombres y mujeres 9 . Sobre este punto, cabe señalar que la industria de la moda ha sido históricamente un área donde se ha "permitido" el acceso de mujeres a puesto de liderazgo de manera más rápida y anterior que en otras industrias.

En efecto, la teoría del denominado "techo de cristal", apunta a que no solamente las empresas deben promover la igualdad de acceso y de salarios a las mujeres respecto de los hombres, sino que éstas también cuenten con las mismas oportunidades de acceder a puestos de toma de decisiones y de poder. ${ }^{10}$

Como consecuencia lógica de los puntos precedentes, la normativa interna de toda empresa, debe ser clara y a la vez drástica al condenar todo tipo de IV) Discriminación al interior de la misma, entendiéndose por ésta toda acción que tenga como criterio de conducta, actos fundamentados en juicios subjetivos (estereotipos y prejuicios) basados en la intolerancia, el rechazo y la ignorancia. Al respecto, toda PDIND debe incorporar sanciones respecto de cualquier acto, omisión o expresión basada en la homofobia, misoginia, xenofobia, segregación racial, antisemitismo, racismo y otras formas conexas de intolerancia que serán entendidas como discriminación.

Las empresas de moda no son ajenas a la tendencia normativa y a la autorregulación. Desde el año 2019 la casa de moda italiana Prada decidió crear un Consejo Asesor en temas de diversidad e inclusión para reflejar en la toma de decisiones de la compañía, los gustos, necesidades y tendencias de las personas de color.

"Prada está comprometida a cultivar, reclutar y retener diversos talentos para contribuir en todos los departamentos de la compañía", aseguró la directora ejecutiva y directora creativa principal de Prada, Miuccia Prada ${ }^{11}$, en un comunicado, lo cual es reforzado en convenios con universidades a través de pasantías con el objetivo de ir reduciendo las brechas de inclusión en la toma de decisiones en la industria de moda.

Otros ejemplos de iniciativas tendientes a maximizar los beneficios de la diversidad en la moda son los que por ejemplo ha tomado la casa Gucci, perteneciente al grupo LVMH. 
"La diversidad es clave para nuestra inteligencia colectiva"12, dijo el martes Francois-Henri Pinault, presidente de la matriz de Gucci. Al respecto, las medidas a tomar por el conglomerado francés, pasan -por ejemplo-, en reestructurar que los diseñadores jefes de los grupos creativos dejen de ser exclusivamente de raza blanca.

Es justamente lo que está ocurriendo en las principales casas de alta moda europea, lo que se pretende lograr en un futuro con todas las empresas de moda: Que no solamente se adopte la autorregulación interna en materia de códigos de conducta o políticas corporativas, sino que, además, esas decisiones pasen a formar parte de la política corporativa de cara a los clientes y consumidores.

Ya en pleno siglo XXI, marcas como Mango, Tommy Hilfiger ${ }^{13}$, Miu Miu, Prada, H\&M, Valentino, Cavalli o Levis han adoptado una política que desde hace décadas tímidamente comenzó a adoptar la italiana Benetton: Apostar por la diversidad en su propuesta, principalmente a través de modelos diferentes en cuanto a razas, edades y nacionalidades. Hoy esa tendencia ha incluido diferentes tipos de cuerpos, marcados igualmente por la tendencia del Body Positive, aquel movimiento global que busca la aceptación del cuerpo -más allá de estándares publicitarios que en la mayoría de los casos obedecen a patrones caucásicos-, y que cada día gana más adeptos en el mundo. ${ }^{14}$

Cada vez nos alejamos más de los estándares que hasta comienzos del presente siglo dominaron la publicidad en el mundo. A lo ya señalado, agregar que publicidad con modelos de tallas grandes -o Plus Size-, es cada vez más natural y generalizada no solamente en marcas de retail masivo, sino que también en casas de alta moda. Similar tendencia estamos viendo con modelos hombres y mujeres por sobre los 50 años, en una lógica bastante sensata, tomando en cuenta la curva ascendente al promedio de edad y al envejecimiento de la población.

Atrás van quedando los tiempos donde la perfección -estilo "ángeles de Victoria's Secret"-, dominaban las pasarelas y la publicidad. No es casualidad entonces que dicha marca haya cancelado indefinidamente su tan conocido show o desfile de modas en noviembre de 2019, anunciando una evolución en la marca. ¿Las razones? Tal como lo señala el diario El País de España, "la caída de audiencias, las pérdidas económicas, el Me Too y la llegada nuevas firmas han hecho que la célebre casa de lencería tenga que replantearse su estrategia y colgar las alas"15.

\section{Conclusiones}

Es importante señalar que el Derecho establece parámetros, lineamientos mínimos de conducta y que somos las personas -y en este caso las organizaciones-, las llamadas a mantener vivas las políticas corporativas. Una empresa no puede quedarse satisfecha implementando una PIDND que simplemente "cumpla con la ley" o que permita que personas diversas trabajen en la compañía. Lo anterior además de mezquino sería ineficaz. La práctica de los lineamientos normativos es la que hace que el sistema "respire". Por ejemplo, en la filial española del grupo Novartis, se ha implementado un plan de mentores inversos, donde un empleado junior se convierte en el tutor de uno más antiguo, de mane- 
ra inversa a la habitualidad de los casos. "Es muy inspirador para los dos. Para la persona más joven, porque habla regularmente con algún líder de la organización, y para el líder también es muy útil. Lo reta, lo cuestiona, le hace plantearse cosas de manera diferente", señala la ejecutiva Begoña Gómez. ${ }^{16}$

Cuando las empresas gestionan el cambio hacia una política activa de diversidad e inclusión, no es solo porque es más justo o beneficia la imagen reputacional de la misma. También es más lucrativo. Tal como señala la revista Vogue Business España:

Un estudio que llevó a cabo Boston Consulting Group el año pasado, midiendo las estadísticas de 1.700 empresas de distintos tamaños y sectores en todo el mundo, concluyó que aquellas que tienen equipos de liderazgo más diversos incrementan sus ganancias hasta un $19 \%$, debido sobre todo a un vector crucial: la innovación. La fricción entre diferentes lleva a nuevas soluciones.

El ejemplo inverso lo vemos en Victoria's Secret. En el mundo actual, la empresa que no se adapta a los cambios y distintos movimientos (\#MeToo, Body Positive, LGTBI) se encuentra prácticamente destinada a morir. Los consumidores castigan a quien no se adapta a la diversidad y a generar una oferta mayor para los distintos tipos de realidades, cuerpos, colores, razas o géneros.

Victoria's Secret ha tratado de revertir la situación: Otorgar protagonismo a modelos de tallas grandes, incorporar a la maniquí con vitíligo Winnie Harlow e incluso utilizar publicidad con fotografías sin retoques o Photoshop. Pero lamentablemente un nuevo perfil de consumidores y la irrupción de nuevas marcas, complican al "paraíso" de los ángeles y cuerpos perfectos. Como concluye la nota de prensa: "(...) lo que ha terminado de rematar a la marca han sido los nuevos tiempos. El Me Too, el cambio de paradigma, la aceptación de todos los cuerpos, el ir más allá del 90-60-90... ha supuesto la puntilla para los cuerpos supuestamente perfectos de Victoria's Secret."17

De acuerdo a lo anterior, la relevancia jurídica de gestionar la diversidad e inclusión al interior de una empresa de moda, no puede quedarse justamente en eso: Una moda. Por el contrario, sobre este punto, Karina Cisterna, Directora Ejecutiva de AHA Inclusión \#SoyPromociona, establece los lineamientos para construir una estrategia corporativa inclusiva $^{18}$, los que apuntan principalmente a establecer un propósito común; medir la "línea base", es decir, realizar un levantamiento de cuan diversa es la organización en el punto de partida; y generar un plan de acción colaborativo, el cual considere a la inclusión y diversidad como un proceso complejo dentro de la organización, que va más allá de declaraciones de principios y diagnósticos, sino que principalmente acompañado de compromiso, legitimidad de los instrumentos normativos e indicadores de medición objetiva del éxito de la misma.

Sin duda un tema entretenido y de gran relevancia, no solo jurídica, sino que sin duda económica. La diversidad e inclusión deben partir en toda empresa -de moda o no-, como una forma de autorregulación normativa en temas de cumplimiento corporativo interno. Lo anterior otorga un valor reputacional mayor a aquellas empresas que establecen principios de respeto y promoción a la diversidad.

En el caso de las empresas de moda, el desafío es mayor. Además de cumplir con aspectos 
de relación interna, deben promover externamente, a modo de imagen y marca corporativa, principios y valores que incorporen nuevos estándares y conceptos de belleza y moda inclusiva, de acuerdo a los nuevos tiempos.

El desafío de la creación y difusión normativa es de los abogados. La planificación y el éxito de la imagen de la marca será, de los responsables de marketing.

\section{Notas}

1. En el caso chileno, los preceptos constitucionales que garantizan la igualdad los podemos distinguir en dos artículos distintos. El artículo $1^{\circ}$ de la Carta Fundamental señala que "las personas nacen libres e iguales en dignidad y derechos". Por su parte, el artículo 19 -que da inicio al catálogo de derechos del Capítulo III-, señala en su enunciado que "la Constitución asegura a todas las personas (...) $2^{\circ}$ La igualdad ante la ley. En Chile no hay persona ni grupo privilegiados. En Chile no hay esclavos y el que pise su territorio queda libre. Hombres y mujeres son iguales ante la ley. Ni la ley ni autoridad alguna podrán establecer diferencias arbitrarias."

2. El 24 de julio de 2012 fue publicada en el Diario Oficial de Chile la ley 20.609, que establece Medidas contra la discriminación, también denominada "ley Zamudio", en honor al joven Daniel Zamudio, tristemente asesinado por un grupo de jóvenes "neonazis" en un crimen de características homofóbicas ocurrido en un céntrico parque de Santiago. En su artículo $2^{\circ}$ establece que "para los efectos de esta ley, se entiende por discriminación arbitraria toda distinción, exclusión o restricción que carezca de justificación razonable, efectuada por agentes del Estado o particulares, y que cause privación, perturbación o amenaza en el ejercicio legítimo de los derechos fundamentales establecidos en la Constitución Política de la República o en los tratados internacionales sobre derechos humanos ratificados por Chile y que se encuentren vigentes, en particular cuando se funden en motivos tales como la raza o etnia, la nacionalidad, la situación socioeconómica, el idioma, la ideología u opinión política, la religión o creencia, la sindicación o participación en organizaciones gremiales o la falta de ellas, el sexo, la maternidad, la lactancia materna, el amamantamiento, la orientación sexual, la identidad de género, el estado civil, la edad, la filiación, la apariencia personal y la enfermedad o discapacidad."

3. El artículo $5^{\circ}$ del Código del Trabajo, establece que "El ejercicio de las facultades que la ley le reconoce al empleador, tiene como límite el respeto a las garantías constitucionales de los trabajadores, en especial cuando pudieran afectar la intimidad, la vida privada o la honra de éstos". La tutela de derechos fundamentales es un procedimiento cautelar cuya titularidad activa la tienen todos los trabajadores y el sindicato, consistente en que "el trabajador afectado directamente o el Sindicato, invocando un derecho o un interés legítimo, podrán denunciar ante la Inspección o ante los Tribunales de Justicia la vulneración de un derecho fundamental o una práctica antisindical o desleal en la negociación colectiva. (Fuente: https://www.dt.gob.cl/portal/1628/w3-article-103242.html).

4. Comúnmente se ha entendido como Soft Law a un conjunto de normas, principios o instrumentos, que si bien carecen de rango normativo en sentido estricto -por cuanto no 
emanan de instituciones de carácter legislativo propiamente tal-, pero que condicionan en cierta medida la soberanía jurídica de los estados, obligándose éstos a cumplir con dichas normas, principios o instrumentos.

5. La ley mercante o ley del comerciante, del latín lex mercatoria, fue inicialmente un sistema jurídico utilizado por los comerciantes en la Europa medieval. Por medio de este conjunto de normas y principios, establecidos por los propios comerciantes, éstos regulaban sus relaciones.

6. Con un desarrollo reciente, la lex sportiva o Derecho del Deporte dice relación con el conjunto de normas de carácter local o internacional aplicadas a la regulación de la práctica y contratación deportivas, tanto en lo relativo a reglas propias de cada disciplina como a usos y jurisprudencia de disputas generadas en el mundo del deporte.

7. En este capítulo seguimos las ideas de la profesora Isabel Turégano Mansilla, tanto las contenidas en su obra "Justicia global, justicia legal e imperio de la ley (Biblioteca Virtual Miguel de Cervantes, Alicante, 2017), como también las expresadas por la autora en su exposición de 12 de enero de 2018 en el curso de Derecho público global, parte de XIX Programa de postgrados de derecho de la Universidad de Castilla La Mancha, Toledo.

8. https://business.vogue.es/tendencias/articulos/diversidad-inclusion-empresas/209

9. En Chile, la ley 20.348, publicada en el Diario Oficial con fecha 19 de junio de 2009 resguarda el derecho a la igualdad en las remuneraciones entre hombres y mujeres. Su artículo 1 introduce un nuevo artículo 62 bis al Código del Trabajo, señalando que "El empleador deberá dar cumplimiento al principio de igualdad de remuneraciones entre hombres y mujeres que presten un mismo trabajo, no siendo consideradas arbitrarias las diferencias objetivas en las remuneraciones que se funden, entre otras razones, en las capacidades, calificaciones, idoneidad, responsabilidad o productividad."

10. Para la Comisión Nacional de Investigación Científica y Tecnológica de Chile-CONICYT-, "Se denomina así a una superficie superior invisible en la carrera laboral de las mujeres, difícil de traspasar, que nos impide seguir avanzando. Su carácter de invisibilidad viene dado por el hecho de que no existen leyes ni dispositivos sociales establecidos ni códigos visibles que impongan a las mujeres semejante limitación, sino que está construido sobre la base de otros rasgos que por su invisibilidad son difíciles de detectar." En: https://www.conicyt.cl/blog/2009/06/02/el-techo-de-cristal/

11. https://www.expoknews.com/diversidad-e-inclusion-en-la-moda-prada-las-impulsara/

12. Ídem

13. La marca Tommy Hilfiger creó la línea Tommy Adaptive, línea inclusiva para personas con discapacidad. https://usa.tommy.com/en/tommy-adaptive

14. Interesante nota al respecto en el siguiente enlace: https://www.elmostrador.cl/braga/2017/11/08/body-positive-el-movimiento-mundial-que-busca-la-aceptacion-delcuerpo-y-que-se-vuelve-cada-vez-mas-popular/

15. https://elpais.com/elpais/2019/11/22/gente/1574412098_714456.html

16. https://business.vogue.es/tendencias/articulos/diversidad-inclusion-empresas/209

17. https://elpais.com/elpais/2019/11/22/gente/1574412098_714456.html

18. Karina Cisterna: "Diversidad e Inclusión laboral: De la moda al impacto en el negocio", en https://www.df.cl/noticias/opinion/columnistas/diversidad-e-inclusion-laboralde-la-moda-al-impacto-en-el-negocio/2019-08-22/143251.html 
Abstract: The influence of Soft Law on the self-regulation mechanisms of companies and their contribution to the State's regulatory action on certain issues. The importance of incorporating in the codes of conduct or corporate policies, norms on the promotion of diversity and inclusion and prohibition of discrimination, in particular in fashion and / or retail companies.

Keywords: Soft Law - Self-regulation - Codes of Conduct - Corporate Policies Diversity Inclusion - Rights - Fashion Companies - Corporate Policy.

Resumo: É analisada a influência do Soft Law nos mecanismos de autorregulação das empresas e sua contribuição para a ação regulatória do Estado em determinadas questões. É apresentada a importância de incorporar nos códigos de conduta ou nas políticas corporativas normas sobre promoção da diversidade e inclusão e proibição de discriminação, principalmente em empresas de moda e / ou varejo.

Palavras chave: Soft Law - Auto - regulação - Códigos de Conduta - Políticas Corporativas Diversidade - Inclusão - Direitos - Empresas de Moda - Política Corporativa.

[Las traducciones de los abstracts fueron supervisadas por el autor de cada artículo] 